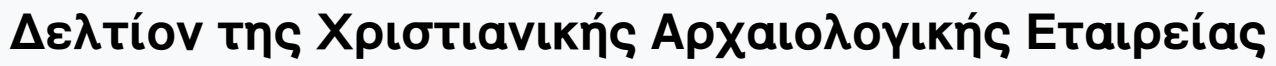

Tó 11 (1983)

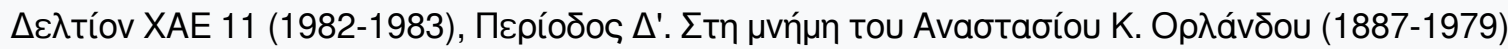

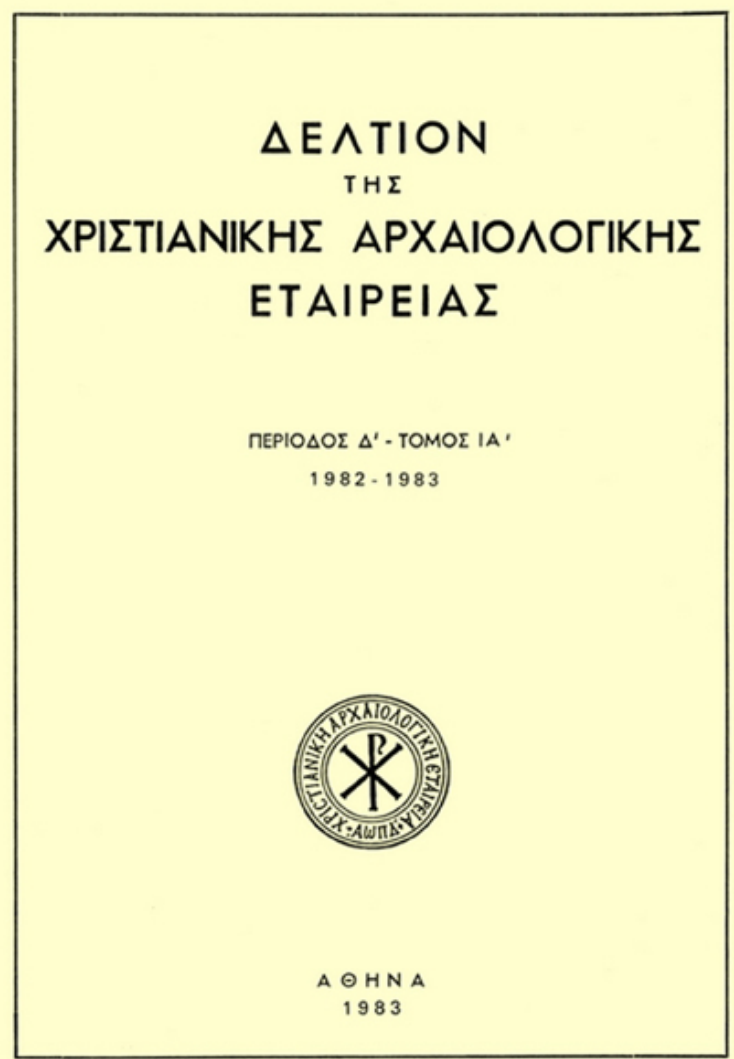

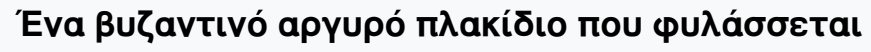

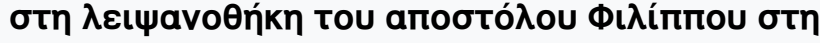
$\Phi \lambda \omega \rho \varepsilon v t i ́ a$

Giovanni CANONIDIS

doi: $10.12681 /$ dchae.936

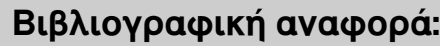

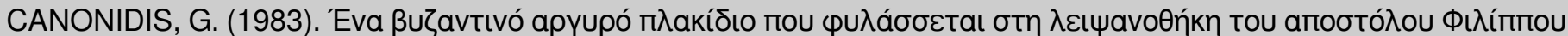

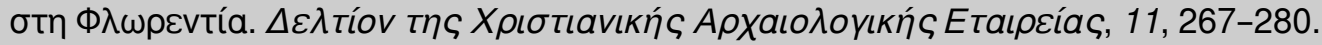

https://doi.org/10.12681/dchae.936 


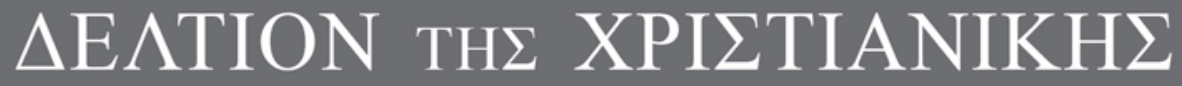 APXAIO $\Lambda$ OГIKH $\Sigma$ ETAIPEIA $\Sigma$}

Una placchetta d' argento bizantina conservata entro il reliquiario di San Filippo Apostolo a Firenze

Giovanni CANONIDIS

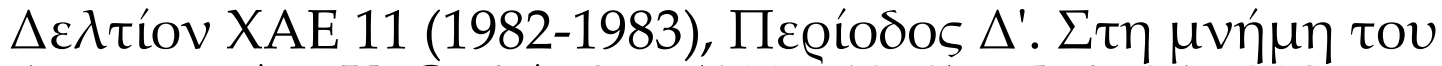

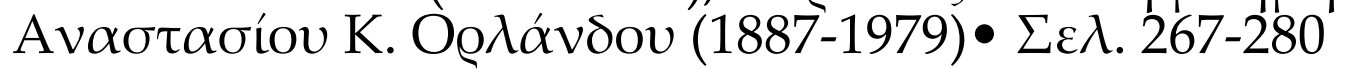

A@HNA 1983 


\title{
UNA PLACCHETTA D' ARGENTO BIZANTINA CONSERVATA ENTRO IL RELIQUIARIO DI SAN FILIPPO APOSTOLO A FIRENZE
}

\author{
Funzione originaria della placchetta, tecnica della sua \\ esecuzione e suo aspetto prima e dopo il restauro del 1954
}

L'oggetto di questo studio è una lamina d'argento bizantina conservata nel Museo dell'Opera del Duomo di Firenze, presa in esame con l'intenzione di giungere a una sua datazione quanto più precisa possibile, spiegare la sua funzione e infine risalire attraverso di essa all'ambiente artistico e culturale che l'ha generata. Le vie scelte per condurre questo esame sono il vaglia delle fonti storico-documentari, lo studio paleografico dell'iscrizione su di essa apparsa e infine l'analisi iconografica e stilistica dell'opera in modo da arrivare a delle conclusioni sulla stessa che abbiano fondamento critico.

Iniziando, quindi, il nostro esame della placchetta raffigurante San Filippo Apostolo si nota che essa è di piccole dimensioni (Fig. 1-2) ${ }^{1}$. L'apostolo è sbalzato in posizione frontale con la mano destra alzata per benedire, mentre nella sinistra stringe un rotolo. La testa giovanile del santo, con i capelli ricciuti, viene incorniciata in una aureola, disegnata da una doppia incisione. Il santo indossa una tunica e un manto con il segmentum ${ }^{2}$ nel braccio destro. Dietro al santo non esiste alcuno sfondo paesaggistico o architettonico. I suoi piedi sono scalzi e non poggiano su alcuna base; non vi è inoltre alcun riferimento ad un suolo su cui potrebbe situarsi la figura.

1. Le misure della placchetta con la figura di san Filippo Apostolo non le conosciamo in quanto non ci è stato possibile misurarla, nè ne sono a conoscienza gli uffici competenti della Soprintendenza di Firenze. La Brunetti che recentemente si è interessata della placchetta di San Filippo Apostolo (L. B e c ch e ruzzi e G. B r un e t t i, Il Museo dell'Opera del Duomo di Firenze, II, Milano 1970, p. 101), non ci dà ulteriori notizie riguardo le dimensioni di essa. Infine gli studiosi che l'hanno precedentemente trattata non citano alcuna notizia sulle misure della stessa.

2. Segmentum: si chiamavano orbiculi quando non portavano ricami, tabulae, tabliae, tablion quando erano quattrangolari e grandi e scutalae quando erano rettangolari. Patagium infine si chiamava il segmentum che si metteva attorno al collo ed era riccamente ornato. Gli artisti non conoscevano bene la funzione di questi e li copiavano casualmente dagli antichi modelli (R. B i a $\mathrm{ch}$ i B a n d ine 1li, Hellenistic-Byzantine Miniatures of the Iliad (Ilias Ambrosiana). Olten 1955, p. 97). 


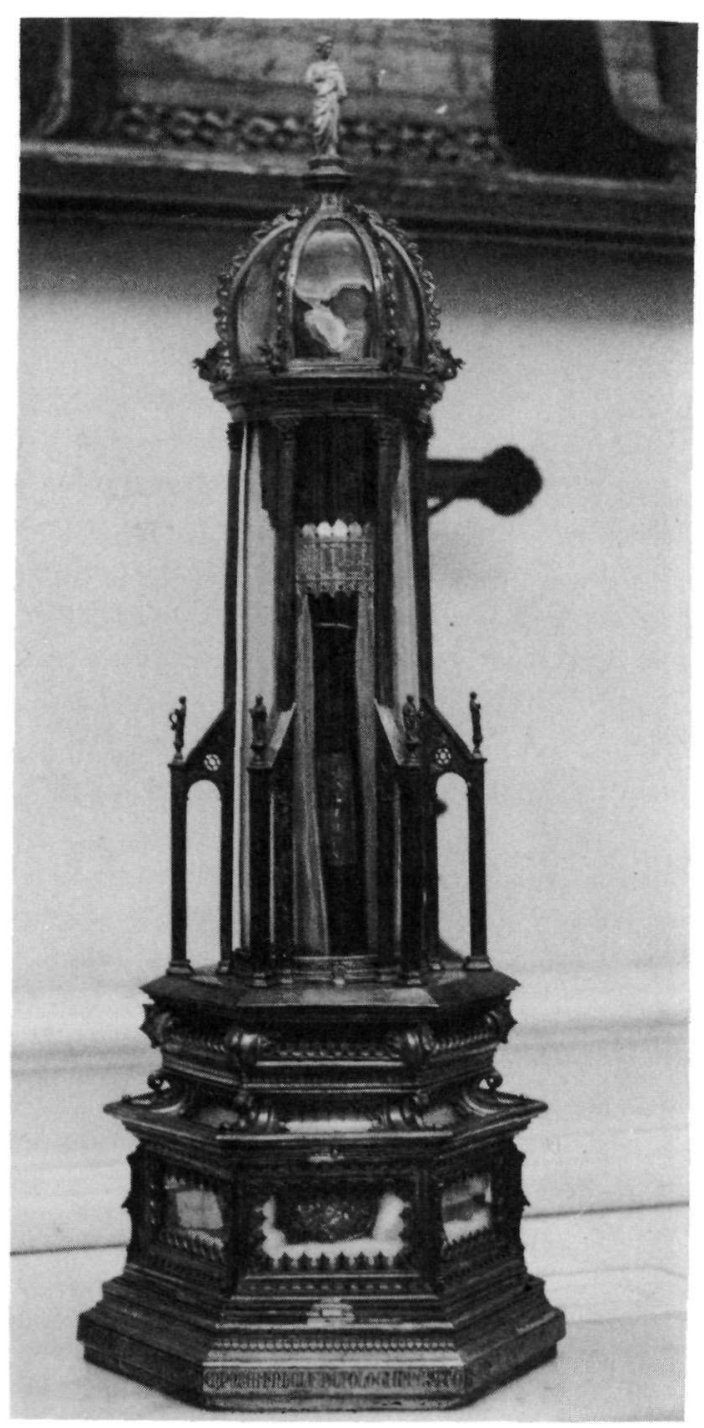

Fig. 1. L'attuale reliquiario di S. Filippo Apostolo oggi collocato nel Museo dell'Opera del Duomo.

Ai lati dell'immagine è inciso verticalmente il nome del santo in caratteri greci. Un cordone perlinato isola la figura; nelle sue parti laterali, al di là del. segmento di separazione, sono degli ornamenti floreali simili nelle due parti, che si sviluppano in senso verticale. Gruppi di quattro petali lanceolate, uniti per le estremità, formano dei rombi; nello spazio tra un petalo e l'altro si trovano delle piccole sfere sbalzate. Tra i motivi ornamentali della parte destra e di quella sinistra non esiste una precisa corrispondenza 
Fig. 2. La placchetta con $\mathbf{S}$. Filippo Apostolo appianata durante il restauro del 1954.

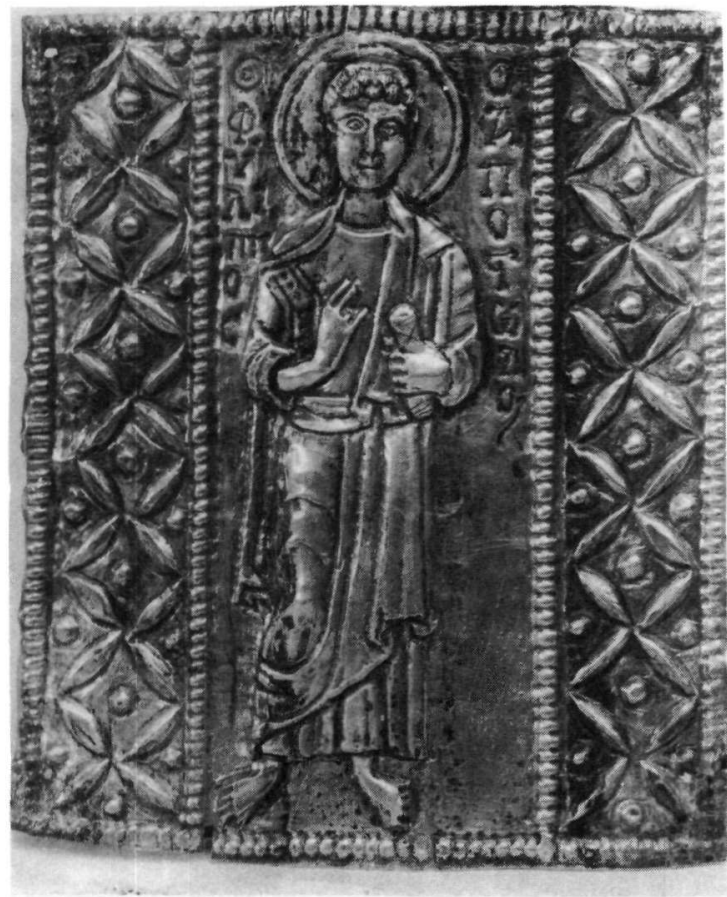

simmetrica, cioè i gruppi dei petali non si collocano in maniera identica nei dui lati. Immediatamente si potrebbe pensare ad un errore da parte dell'orefice, ma poi osservando bene la placchetta si scarta una tale ipotesi. Si può notare infatti una precisa uniformità nel motivo ornamentale dato dalla sua continuità: il disegno della parte destra continua infatti, nonostante la netta separazione, nella parte sinistra. L'unione delle due parti si nota tenendo presente lo schema del rombo formato dai petali: la composizione ornamentale della parte destra comincia con un mezzo rombo, che si conclude nell'altro mezzo con cui inizia la parte sinistra. Il materiale con cui è stata fatta questa lamina è, come si è già detto, argento dorato; essa è stata prima sbalzata e poi finita a cesello.

Un'ampia descrizione delle tecniche dello sbalzo e del cesello nel Medioevo ci è pervenuta grazie alla Diversarum Artium Schedula di Theophilus Presbyter et monachus ${ }^{3}$, dove nel terzo libro viene descritta tutta l'arte

3. Diversarum Artium Schedula scritta da Theophilus presbyter et monachus nel X secolo. L'origine dell'autore di questa Schedula non si può rintracciare con sicurezza; Angelo Lipinsky sostiene presumibilmente un'origine constantinopolitana di esso. La tradizione del testo della Schedula è rimasta legata a dieci manoscritti, dei quali, però tre solo 
della lavorazione dei metalli in novantacinque capitoli; le nostre notizie sul procedimento tecnico dello sbalzo e del cesello non sono però prese direttamente dalla Diversarum Artium Schedula ma da due articoli di Angelo Lipinsky ${ }^{4}$ dedicati ad essa. Il procedimento della lavorazione a sbalzo e cesello teoricamente è abbastanza semplice; il punto di partenza è la preparazione di un'impasto ottenuto mischiando pece greca, polvere di mattone o gesso, cera ed olio. Nella preparazione di questo impasto bisogna tener conto della temperatura stagionale e dell'ambiente che influiscono ambedue sulla sua plasticità; conseguentemente bisognerà aggiungere più gesso d'estate, per renderlo più rigido, e maggior quantità di olio e grasso d'inverno affinchè divenga più plastico.

Questo impasto di plasticità modificabile, viene messo sulla lamina d'oro o d'argento che deve essere lavorata; esso servirà come supporto non rigido per la lamina che attutisca i colpi del martello durante la lavorazione. In seguito, con un martelletto si comincia a picchiare sulla lamina, sbalzandola, tirandola e dandola una prima incurvatura che abbozza le forme del soggetto da rappresentare e che sarà finita in seguito lavorando la lastra sull' altro lato. Durante il lavoro di martellattura il metallo in lavorazione tende ad indurirsi, ragion per cui staccato dalla pece viene posto sul fuoco per restituirgli la malleabilità necessaria. Fatto ciò la lastra viene messa rovesciata sull'impasto di pece per continuare il lavoro sulla facciata opposta picchiandovi sopra con martello e ceselli di varie misure e fogge. Le rifiniture da incidere a solco sull'opera si fanno con bulini e ciappole. Infine, l'opera viene sottoposta ad un bagno sgrassante per togliere ogni traccia di pece e di unto. Con questa tecnica usata soprattuto per lavori in oro e argento può essere lavorato anche il rame.

Per ora fermiamoci alla descrizione elementare della placchetta d'argento e della tecnica, usata per la sua esecuzione, per proseguire con una ricerca su quale significato essa può avere.

Non riteniamo che questa lamina, applicata all'osso del braccio di San Filippo Apostolo, aquisti un particolare significato dal fatto che non riscon-

sono i più antichi ed anche i più autorevoli: I) Codex Gudianus Nr 69 (30 fogli di pergamena). II) Codex Vindobonensis Nr 2527 (117 fogli di pergamena). III) Codex Harleianus $\mathrm{Nr} 3915$ (120 fogli di pergamena).

4. Gli articoli di Ange 10 Li p insk y serviti per la nostra descrizione del procedimento tecnico dello sbalzo e del cesello sono: a) Oreficeria, argenteria e gioieieria nel tardo impero e nell'alto Medio Evo: La Diversarum Artium Schedula di Theophilus presbyter et monachus, sta in Corsi di cultura sull'arte ravennate e bizantina, Ravenna 8-21 marzo 1964, Ravenna 1964. b) Introduzione tecnica all'arte orafa bizantina, sta in Corsi di cultura sull'arte ravennate e bizantina, Ravenna 20 Marzo-1 Aprile 1966, Ravenna 1966. 
triamo altri esempi di placchette rappresentanti figure di santi applicate a reliquie. Molto più consueto ci sembra che venisse applicata sulla reliquia una lamina d'oro o d'argento con il nome del santo solo inciso su di essa come ci dimostra un'esempio nel museo di San Marco a Venezia: la falange del dito di San Cristoforo ${ }^{5}$. Eliminata l'ipotesi che l'attuale uso della placchetta sia quello per il quale fu creata, dobbiamo cercare di determinare ipoteticamente la sua funzione originaria.

Conosciamo dalla fonte principale della storia della traslazione, che la reliquia arrivò a Firenze, e per un certo periodo fu anche custodita entro un forzierino d'argento. Tenendo conto dello spirito religioso di quell'epoca, che permetteva di diventare reliquia qualsiasi cosa venisse in stretto contatto per un periodo con una vera reliquia (reliquia «ex affinitate») ${ }^{6}$, si può supporre che questa placchetta facesse parte originariamente del forzierino che scomparve con la deposizione della reliquia nel nuovo reliquiario. La sua conservazione quindi non si può attribuire ad alcun motivo storico-documentario, ma ad un semplice scrupolo religioso.

Osservando le fotografie fatte durante il restauro del 1954 (Fig. 2-3) ${ }^{7}$, possiamo avere un'idea del modo con cui la placchetta era applicata all'osso del braccio ${ }^{8}$. Nella foto in cui si vede la lamina incurvata in modo da formare un cilindro, si distingue un materiale che unisce gli angoli della plac-

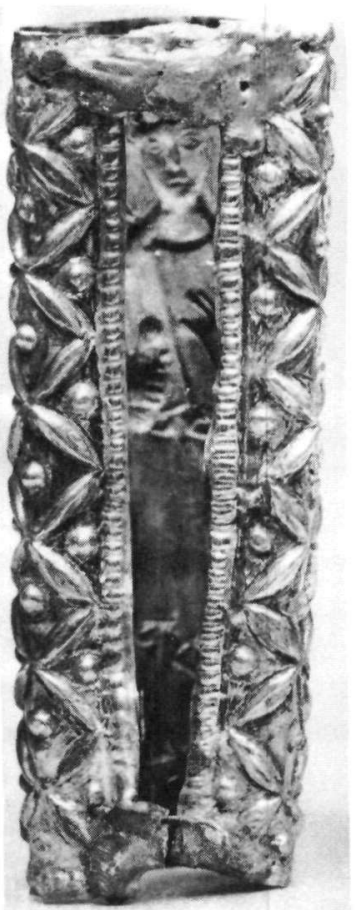

Fig. 3. La placchetta con S. Filippo Apostolo in forma di cilindro prima del restauro del 1954.

chetta. Bino Bini che ha partecipato al restauro ci ha spiegato che si tratta

5. Reliquia-encolpio d'argento del XIV secolo. Foto di questa reliquia si trova nel catalogo Il tesoro di San Marco, a cura di H. Hahloser, Firenze 1971, tav. XXX.

6. H. L e cle r c q nella voce Reliques et reliquaires, che sta in Dictionnaire d'Archéologie chrétienne et de Liturgie, XIV 2, pp. 2294-2359, spiega che poteva diventare reliquia anche un'oggetto che era per un periodo in stretta relazione con una reliquia vera.

7. Il professore Bino Bini ci ha dato un suo breve appunto preso durante il restauro del 1954: «Rifacimento di tutte le montature e di conseguenza anche quella della placca con la figura di San Filippo Apostolo, per fermare tutti i pezzi sulla figura del santo. Alla base addrizzatura. Il restauro si è svolto in tre fasi: addrizzatura, ripulitura, protezione».

8. Non possiamo confermare con precisione che l'applicazione sia l'originale del 1425 , perchè il reliquiario è stato rimaneggiato e restaurato nei periodi posteriori dalla sua costruzione. 
di ceralacca che veniva usata per sigillare le reliquie. Dunque, una volta tagliata probabilmente la scorta di metallo forata che circondava la lamina, servita per la montatura sul legno della placchetta ${ }^{9}$ con dei piccoli chiodini, si sono aperti quattro fori agli angoli della placchetta. Essi servivano per l'applicazione intorno all'osso: passato un filo di metallo due o tre volte attorno alla reliquia, lo facevano passare in seguito anche da questi fori, legavano le punte del filo e poi mettevano la ceralacca e sigillavano la reliquia. Nella fotografia con la lamina in forma di cilindro, però, non si distingue alcun sigillo. Probabilmente le punte dei fili uscivano dai due fori che si vedono a destra della legatura superiore, quindi si faceva il nodo e si metteva il sigillo sulla ceralacca. Il sigillo non si è conservato perchè i restauratori per staccare la placchetta hanno dovuto romperlo, previo permesso della curia. L'uso del filo e della ceralacca per l'applicazione vengono a confermare del primo la traccia di esso che si distingue nella parte inferiore della placca nella fotografia mostrante essa in forma di cilindro, e della seconda il fatto che nella foto della placchetta appianata e ripulita non esiste alcuna parte rovinata. Infatti, se si usasse invece della ceralacca un metallo, risulterebbero gli angoli della lamina rovinati, cosa che non riscontriamo nella foto dopo la ripulitura.

\section{L'iscrizione e analisi iconografica della figura dell'apostolo Filippo}

La prossima indagine sarà l'esame paleografico e iconografico della placchetta, sempre nel nostro tentativo di stabilire l'origine e la data di essa.

Ai lati della figura di San Filippo Apostolo è inciso il suo nome in lettere maiuscole greche: O ФҮАІППОС O AПО $\Omega \Lambda O S$. Anche da questa scritta non ricaviamo però alcun aiuto; infatti la lingua greca era la lingua ufficiale non solo della corte constantinopolitana, ma di tutto l'impero bizantino. Dal punto di vista paleografico inoltre non è possibile stabilire un'ambito cronologico preciso per l'opera; le lettere maiuscole infatti, al contrario di quelle minuscole, non subirono sostanziali mutamenti col passare dei secoli ${ }^{10}$.

Un'altro punto di partenza per raggiungere il nostro scopo sarebbe

9. Probabilmente sbalzate all'inizio separatamente le parti del cofanetto vennero in seguito a ricoprirlo. Esso era di legno e l'applicazione delle lamine d'argento si effettuava con dei piccoli chiodini. Questi chiodini li facevano passare dai fori aperti attorno alle lamine e in seguito li fissavano sul legno del cofanetto. La parte forata servita per la montatura della lamina sul forzierino, con il quale si è trasportata la reliquia di San Filippo, venne probabilmente tagliata perchè non serviva all'applicazione sull'osso del braccio dell'Apostolo Filippo.

10. L'impossibilità di datare la placchetta in un ambito cronologico ristretto per 
l'iconografia, ma anche in questo caso sorgono delle difficoltà. Infatti con un'esame retrospettivo dell'arte bizantina ci accorgiamo della sua fedeltà a una iconografia stabilita, specialmente quando si tratta di rappresentare figure singole di santi. Atteggiamento simile a quello della figura di San Filippo Apostolo presentano per esempio opere che risalgono al VI secolo come alcuni dei profeti rappresentati nella zona media dei mosaici delle pareti laterali di San Apollinare Nuovo a Ravenna. Lo stesso atteggiamento venne lungamente tramandato nell'area artistica bizantina per causa delle sue tendenze conservatrici, cosa che testimoniano opere iconograficamente simili di diversi periodi della sua evoluzione. La stessa posizione frontale e gli stessi movimenti dei profeti ravennati e della figura di San Filippo Apostolo della placchetta fiorentina hanno inoltre le figure di Gregorio Nazianzeno, Gregorio di Nissa e Basilio di una miniatura di un codice delle Omelie di Gregorio Nazianzeno (Parigi, Bibl. Nat., cod. gr. 510, fol. 71v), attribuito al 880-883 ${ }^{11}$, il mosaico del timpano nord di Santa Sofia a Constantinopoli rappresentante San Giovanni Crisostomo, opera del IX secolo ${ }^{12}$, la teoria di santi e San Giovanni Crisostomo del mosaico absidale di Santa Sofia a Kiev datato tra il $1042-1046^{13}$ e tante altre opere.

In quanto ai tipi giovanile e simile di San Filippo Apostolo, questi erano consueti fin dal primo cristianesimo come conferma Maria Letizia Casanova ${ }^{14}$, cosa che non ci permette fare delle attribuzioni sicure. Non resta infine che un'attento esame stilistico per determinare con precisione il periodo storico al cui appartiene l'opera in esame.

\section{Analisi stilistica all'interno delle linee di svillupo dell'arte bizantina}

Osservando con attenzione la figura di San Filippo Apostolo della lamina d'argento ci accorgiamo che esso è leggermente inclinato nella sua parte sinistra ${ }^{15}$. Questa leggerissima inclinazione non è attribuibile ad una debolezza tecnica dell'artista. Si può infatti notare il piegamento della sua

vie paleografiche è stata confermata dal prof. Di Benedetto, dopo una analisi della iscrizione.

11. Per questa datazione cfr. S. D. N e r s e s s i a n, The illustrations of the Homelies of Gregory of Nazianzus, Paris. gr. 510. A Study of Connections between Text and Images, sta in DOP XVI (1962), pp. 195 sgg.

12. V. L a z a rev, Storia della pittura bizantina, édizione italiana rielaborata e ampliata dall'autore, Torino 1967, p. 114.

13. Ivi, p. 153.

14. Maria Letizıa Cas a nova, Iconografia di San Filippo Apostolo, sta in Bibliotheca Sanctorum, V, pp. 711-719.

15. La parte sinistra del santo corrisponde alla destra di chi lo guarda. 
gamba destra come se volesse fare un passo in avanti. Notiamo, inoltre, che le vesti di San Filippo Apostolo non gli cadono pesantemente addosso, nascondendolo, ma il leggero panneggio permette di intravedere le linee del corpo. Più precisamente la tunica e il mantello si svolgono in maniera tale da disegnare pieghe che ci permettono di distinguere il volume del corpo: il ginocchio destro è evidentemente flesso. Da tutto ciò risulta un'equilibrata coesistenza delle vesti e del corpo. Da queste prime caratteristiche distinte nell'immagine di San Filippo e dopo una prima anche superficiale analisi stilistica si può attribuire la placchetta nel periodo tra la Rinascenza Macedone ${ }^{16}$ (IX-X secolo), e l'inizio del tredicesimo secolo. Tenendo conto del limite cronologico posto dalla documentazione fornitaci riguardo l'arrivo del braccio di San Filippo Apostolo a Firenze nell'anno $1204{ }^{17}$ e concentrando in seguito la nostra attenzione sul volto del santo possiamo tentare di precisare la nostra per ora troppo vaga datazione. Infatti, nel viso dell'apostolo Filippo domina un sistema di linee che lo rendono fortemente stilizzato. L'espressione resa attraverso questo sistema di linee, è particolarmente austera e astratta. Anche sulla base di questi pochi elementi, aggiunto anche l'inespresso elemento spaziale, ci sembra abbastanza verosimile proporre fin da ora un'attribuzione della placchetta nel XII secolo per il quale Viktor Lazarev, in un discorso sulle miniature del medesimo secolo, dà la seguente definizione dello stile pittorico bizantino:

16. «Rinascenza Macedone» fu chiamata da $\mathrm{Kurt}$ We it z man n l'arte del X secolo, caratterizzata come una fase dell'arte bizantina in cui viene riscoperta l'arte antica. La sua tesi apparve nel suo articolo Der Pariser Psalter MS. Grec. 139 und die mittelbyzantinische Renaissance (sta in Jahrbuch für Kunstwissenschaft 6 (1929), pp. 178-194) e secondo questa una serie di opere, fino allora considerate come espressioni di una continua tradizione ellenistica sul suolo dell'impero romano orientale, vengono esaminate come un segno di una tarda ripresa di questa tradizione dopo la parentesi dell'Iconoclastia durata dal 726 all' 843.

La sua tesi sulla riscoperta classica della «Rinascenza Macedone» impegnò Weitzmann a definire il ruolo che l'arte classica aveva avuto all'interno dell'arte bizantina. $\mathrm{Nel} 1954$ esce così Das klassische Erbe in der Kunst Konstantinopels, sta in Alte und Neue Kunst 3 (1954), pp. 41-59; nel 1966 The Classical in Byzantine Art as a Mode of Individual Expression, sta in Byzantine Art, an European Art, Ninth Exhibition held under the auspices of the Council of Europe, Lectures, Athens 1966, pp. 149-177. La tesi di Weitzmann sulla «Rinascenza Macedone» non fu accettata da tutti gli studiosi; tra i maggiori sostenitori di una tesi diversa alla sua furono: Charles Rufus Morey, Viktor Lazarev e Cyril Mango.

17. La fonte principale delle notizie riguardo la traslazione del braccio di San Filippo Apostolo ci da il Martirologio dell'Archivio dell'Opera di Santa Maria del Fiore, Mores et consuetudines canonice florentine. Passio Reparatae legendae S. Zenobi, Eugenii et Crescenti Bracchii S. Filippi. Nr 20, serie I. 
«Nell'insieme lo stile delle miniature del XII secolo rappresenta il punto culminante nella evoluzione delle tendenze astratte della pittura bizantina» ${ }^{18}$.

L'apparirsi di questo stile nell'ambito dell'arte bizantina non avvenne subito con l'inizio del dodicesimo secolo, ma verso la fine dell'undicesimo. Infatti nell'ultimo quarto dell'XI secolo la maniera fortemente spiritualistica che fiorì durante il suo percorso, cominciò a cedere a forme più astratte con il dominare degli elementi lineari su quelli pittorici. La tendenza alla schematizzazione andava piano piano accenduandosi fino a prendere il sopravvento verso la metà del XII secolo con il risultato di trovarci di fronte ad un'arte di forme piatte, di composizioni d'impronta artigianale, di figure con movimenti di repertorio limitato che si ripetono spesso, di sfondi paesaggistici e architettonici che sono talmente semplificati da perdere ogni legame con la realtà.

Nonostante però questo scadimento dell'espressione artistica nel XII secolo non mancano artisti che producono opere di elevata qualità, dandoci ammirevoli esemplari delle loro capacità. Questi sono secondo Viktor Lazarev gli artisti dei due mosaici della tribuna sud di Santa Sofia raffiguranti l'uno Giovanni Comneno e la sua consorte Irene ai lati della Vergine col Bambino e l'altro la Deesis di cui si sono conservate solo le parti superiori delle figure; ad essi va aggiunto l'artista principale delle maestranze che eseguirono il ciclo di affreschi nella chiesa di San Pantaleimone a Nerezi in Macedonia ${ }^{19}$. Questi capolavori del XII secolo sono secondo Viktor Lazarev espressioni dell'area artistica constantinopolitana che aveva il sopravvento su tutte le altre scuole provinciali dell'impero bizantino. La fine di questa fase non avvenne in un preciso momento storico, ma gradualmente con l'inizio del manifestarsi di nuove regole artistiche nelle opere di avanguardia della fine del XII secolo ${ }^{20}$. Questo nuovo orientamento dell'arte bizantina venne in seguito elaborato e maturato a Nicea che sostituì come capitale Constantinopoli dopo la caduta dell'ultima nelle mani dei crociati nel 1204.

La nostra attribuzione della placchetta con San Filippo Apostolo al XII secolo e la descrizione di questa fase alla quale essa appartiene in base agli studi sulla pittura bizantina sono stati fatti dopo un nostro tentativo di rintracciare una storia sullo sviluppo dell'oreficeria bizantina, che risulta però inesistente nella storiografia moderna nonostante la sua importanza in

18. V. L a z a rev, Storia della pittura bizantina, p. 192 .

19. La chiesa è stata eretta nel 1164 per ordine di Alessio Comneno secondo la scritta greca al di sopra della porta del nartece.

20. Questi sono secondo V. L a z a rev (cit., p. 275), gli affreschi della cattedrale di San Demetrio a Vladimir e le miniature del secondo Vangelo di Dzruci a Tbilisi. 
seno alla società bizantina. La sopracitata scarsezza di documentazione però non significa inesistenza assoluta di opere di oreficeria del periodo che interessa la nostra indagine. Contrariamente opere esistono in numero elevato, ma sono inconsigliabili per i nostri confronti perfino quelle sulle quali vengono anche riprodotte figure singole di santi in posizione frontale. Infatti a causa del forte legame alla tradizione degli artisti bizantini, succede che spesso lo stile dei vari modelli resta discernibile o può perfino dominare sullo stile dell'artista ${ }^{21}$. Non possono così essere considerati come confronto stilistico al fine di una datazione della placchetta fiorentina la figura frontale di San Demetrio di Salonicco sull'omonimo reliquiario custodito nel convento di Vatopedi sul Monte Athos, e la figura dello stesso santo del reliquiario della cattedrale di Halberstadt, ambedue attribuite al XII secolo ${ }^{22}$. Infatti queste due opere presentano diversità stilistiche dalla figura di San Filippo Apostolo dovute molto probabilmente al summenzionato motivo. L'eliminazione di questi due ultimi lavori d'oreficeria per i sopracitati motivi e l'impossibilità di trovarne altri nello stesso materiale adatti per un confronto con la lamina raffigurante San Filippo Apostolo, necessariamente ci obbligano a servirci per il nostro scopo di opere in materiali artistici differenti.

Una conferma della datazione attribuita alla placchetta in esame ci offre un'opera attribuita allo stesso XII secolo. Si tratta di una scultura che si trova nella cattedrale di Caorle vicino a Venezia e rappresenta a rilievo San Agathonico. La datazione al XII secolo proposta convincentemente da Andrè Grabar:

«Mais le style des draperies, amples et larges, où les plis ne sont indiqués que par quelques incisions, est bien dans le gout du XIIe siècle qui cherche à simplifier les surfaces sculptées. La même tendance se manifeste dans le contour général de la figure qui s'élève d'un seul bras au-dessus du fond et s'arrête à peu près partout à la même hauteur. Autrement dit la figure reste plate et ne s'arrondit que vers les bords» ${ }^{23}$.

Il santo viene rappresentato in posizione di orante. Nonostante la lavorazione grottesca del suo corpo in confronto al corpo di San Filippo Apostolo, si riesce ad intravedere sotto il manto la gamba destra del santo che è flessa nel suo tentativo di fare un passo in avanti, cosa che si distingue anche sulla figura dell'apostolo Filippo. Osservando i volti dei due santi si aumen-

21. Su questo problema cfr. soprattutto $\mathrm{K}$. W e it $\mathrm{z} \mathrm{m} \mathrm{a} \mathrm{n}$, Illustration in Roll..., cap. IV: The effect of the concept of the cyclic unit on stylistic criticism, pp. 199-205.

22. A. Grabar, Sculptures byzantines du Moyen Age, Démétrios et le martyrium du saint à Salonique, sta in DOP 5 (1950), pp. 1-28.

23. A. G r a b a r, Sculptures byzantines du Moyen Age (XIe-XIVe siècle), Paris 1976, scheda 125, p. 124. 
tano le caratteristiche comuni delle due opere. Infatti, gli occhi, il naso e la bocca sono disegnati con le stesse linee semplici dando alle immagini una espressione astratta. A questi ultimi elementi comuni si aggiungono oltre, il modo stilizzato dell'esecuzione dei capelli e l'inesistenza di uno sfondo architettonico e paesistico.

Le stesse caratteristiche con le due opere già confrontate presenta inoltre un filone di opere d'arte a partire dalla fine dell'XI secolo fino gli inizi del XIII. Infatti nelle immagini dei quattro santi guerrieri San Demetrio, San Teodoro, San Giorgio e San Procopio di una placca in steatite collocata nel museo del Louvre (Fig. 4) ${ }^{24}$ e quella di San Pantaleimone a mezzo busto di un'altra placchetta in steatite della Walters Art Gallery di Baltimora (Fig. 5) ${ }^{25}$, appartenenti nel periodo tra la fine dell'XI e l'inizio del XII secolo dimostrano chiaramente l'orientarsi verso una schematizzazione dell'arte bizantina. Questa schematizzazione col risultato di una elevata astrazione, si riscontra in opere come la nostra figura di San Filippo Apostolo, in un affresco murale rappresentante San Pantaleimone nella chiesa di San Giorgio a Kurbinovo ${ }^{26}$, in un dipinto murale raffigurante San Pantaleimone nella chiesa degli Agioi Anargyroi a Kastoria ${ }^{27}$, nei resti di una pittura murale della chiesa di Santo Stefano a Kastoria raffiguranti i santi Kosmas e

24. La placchetta in steatite consiste in due parti, così oltre i quattro santi guerrieri della zona inferiore viene rappresentata in quella superiore la Etoimasia. La placchetta apparteneva in origine alla collezione della Comtesse de Bearn, ora nel museo del Louvre. La sua ultima datazione alla fine dell'XI secolo è stata fatta da A. Bank, in base di dati paleografici (A. B a n k, Les stéatites essai de classification, méthodes des recherches, sta in Corsi di Cultura sull'arte ravennate e bizantina, Ravenna, 8-21 marzo 1970, Ravenna, 1970 , p. 361).

25. Cenni sugli elementi in cui è basata la datazione della placchetta in steatite raffigurante San Pantaleimone a mezzo busto, attribuita alla fine del XI seccilo e appartenente alla Walters Art Gallery di Baltimora, non ci sono forniti dalla fonte relativa ( $\mathrm{K}$. We it z mann, Catalogue of the Byzantine and Early Medieval antiquities in Dumbarton Oaks Collection, Ivories and steatites, Washington 1972, p. 97).

26. La datazione dell'affresco con l'immagine di San Pantaleimone di San Giorgio

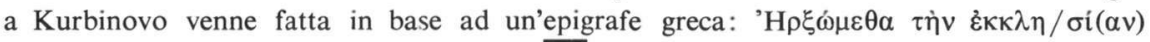

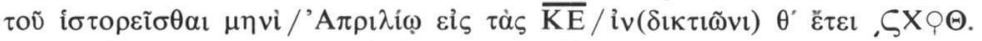

(Abbiamo cominciato di decorare la chiesa il 25 del mese di aprile, nona indictiona dell'anno 6699-1191) (T. M a $1 \mathrm{~m} \mathrm{qu}$ is t, Byzantine 12th century Frescoes in Kastoria. Agioi Anargyroi and Agios Nicolaos tou Kasnitzi, Uppsala 1979, p. 124).

27. La datazione della figura di San Pantaleimone dell'affresco degli Agioi Anargyroi a Kastoria venne fatta da T. Malmquist, la quale sostiene che il gruppo dei santi Kosmas, Damiano, Pantaleimone e Hermolaos: "These are the most common saints in the group, and at least some of them are represented in the 12th century churches. They often hold a medical box, some instruments or bottles in their hands, or simply a martyr's cross» (T. M a $1 \mathrm{mqu}$ is t, cit., p. 90). 
Damiano ${ }^{28}$, nel dipinto su seta con San Giusto nella cattedrale di Trieste ${ }^{29}$ e infine nel volto di San Demetrio di Salonicco ${ }^{30}$ di una icona bizantina appartenente alla Tretiakov Galerie di Mosca, opera attribuita alla fine del XII secolo. In tutta questa serie di opere si può distinguere l'inizio, il cul-

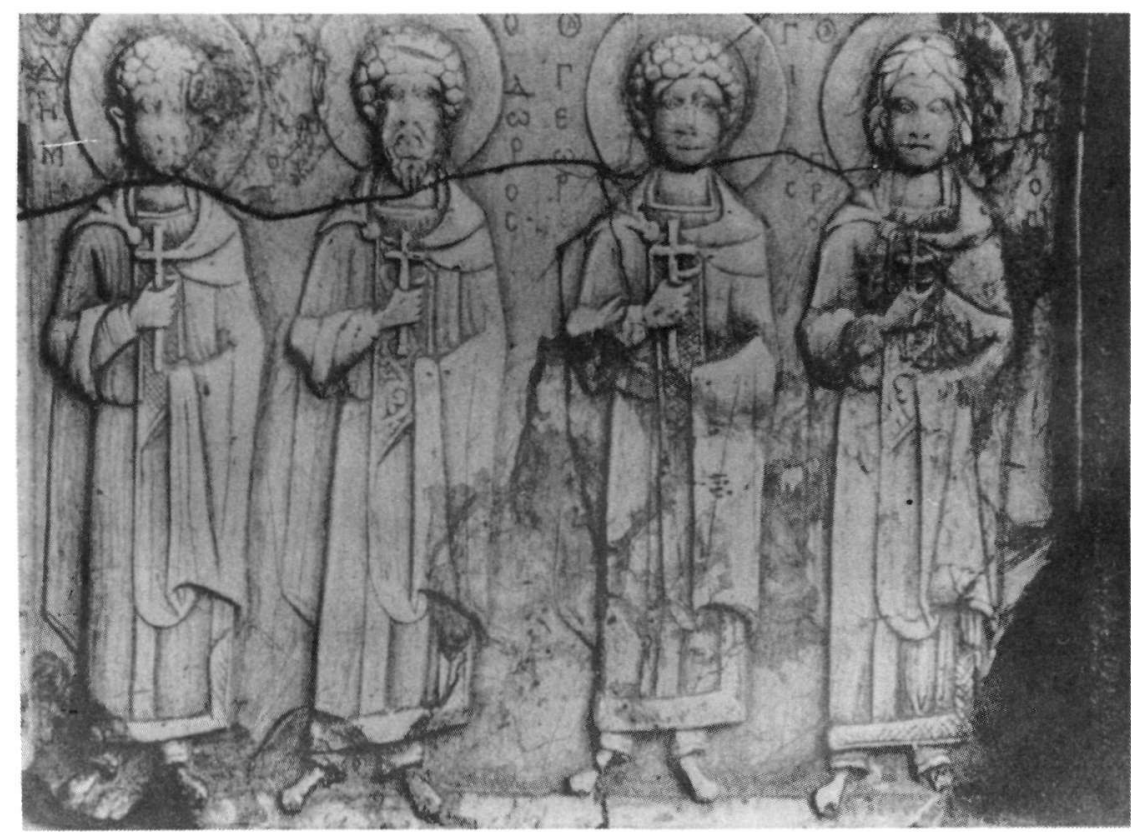

Fig. 4. Museo del Louvre. San Demetrio, San Teodoro, San Giorgio e San Procopio.

mine ed il tramonto di uno stile ben preciso che occupa all'interno delle linee di sviluppo dell'arte bizantina il periodo tra la fine dell'undicesimo secolo e gli inizi del XIII.

28. La fonte relativa delle nostre notizie sull'affresco rappresentante i santi Kosmas e Damiano della chiesa di Santo Stefano a Kastoria non menziona in base quali elementi

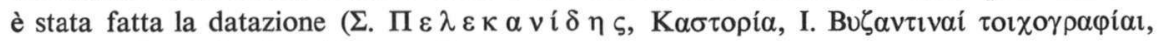

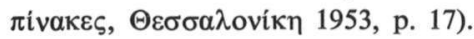

29. Il santo è in posizione frontale con una mano alzata mentre nell'altra tiene una foglia di palma. La parte inferiore del dipinto risulta rovinato. Neanche di questa opera le fonti delle nostre informazioni forniscono in qual modo è stata fatta la datazione (W. F e li cet t i - L i e b e n fels, Geschichte der byzantinischen Ikonenmalerei, OltenLausanne 1956, p. 46).

30. Limitiamo il confronto solo nella parte del viso perchè solo questo è stato salvato dalle ridipinture posteriori subiti dall'opera (W. Felicetti-Liebenfels, cit., pp. 45-46). 


\section{Origine della placchetta}

Dopo l'analisi stilistica e la proposta di una datazione all' interno del XII secolo rimane il problema della definizione dell'ambiente artistico al cui appartiene la placca d'argento che appare però abbastanza problematico.

Fig. 5. Walters Art Gallery di Baltimora. San Pantaleimone.

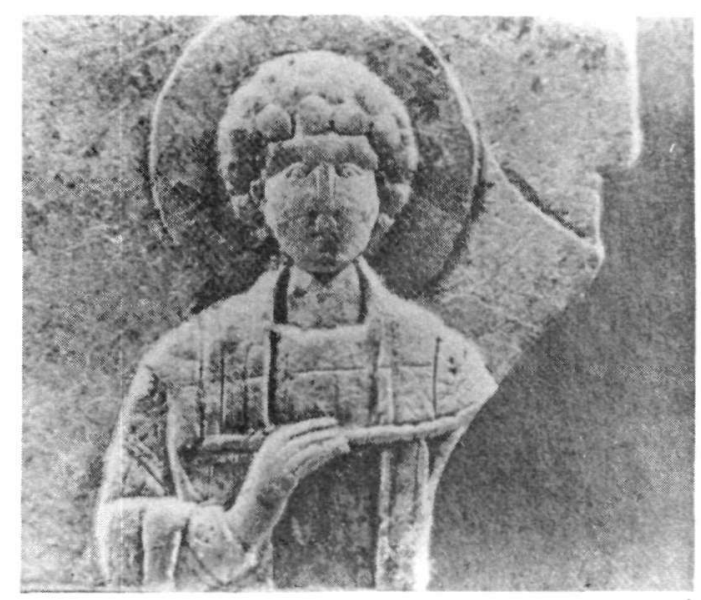

Nelle «Memorie» Istoriche di Giovanni Mariti si fa cenno di un reliquiario entro cui venne portata la reliquia dell'apostolo Filippo da Constantinopoli a Gerusalemme:

«Si ricordava il nostro Patriarca che fino dai tempi in cui Maria Comnena venne sposa in Soria del Re Almerico di Gerusalemme, cioè nel 1168, aveva fra le altre ricchezze portato seco anche un ricco Reliquiario con entrovi un Braccio di San Filippo Apostolo dono che a lei aveva fatto il suo zio l'imperatore Manuel Comneno» ${ }^{31}$.

Nel Martirologio che contiene l'Atto della traslazione del braccio di San Filippo Apostolo, su cui il Mariti basa la sua descrizione, non esiste riscontro a tale notizia; il testo latino non menziona infatti alcum reliquiario:

«Hic siquidem sciebat quid prenominatus imperator hemmanuel largitus fuerat sancti apostoli philippi brachium nepti sue curie marie tempore quo eam tradidit regi almarico in uxorem. Unde tam ipsam quam filiam eiusdem curie marie reginam isabellam uxorem ermerici regis qui de cipro in accon venerat ad regnandum fuit taliter allocutus Filie hieruslem regali diadimate vobis terrenus imperator ex amore precipuo et gratia spirituales apostoli

31. G. M a riti, Memorie Istoriche di Monaco de Corbizzi Fiorentino Patriarca di Gerusalemme, Firenze 1781, p. 48. 
scilicet philippi brachium quod etsi vobis liceat non tamen expedit custodire quia celum celi domino terram autem dedit filiis hominum» ${ }^{32}$.

Un'altra fonte dalla quale Mariti avrebbe ricavato la notizia del reliquiario entro il quale sarebbe stata trasportato il braccio del santo da Constantinopoli a Gerusalemme non ci è pervenuta dal suo lavoro. Contrariamente alla sua abitudine di citare la fonte da cui ha tratto un'informazione, il Mariti non lo fa per questa notizia che riteniamo importante per la nostra ricerca. Dovendo quindi determinare l'area di provenienza della placchetta non possiamo avere un'aiuto dal Mariti. Ci è preclusa la possibilità di supporre che il forzierino d'argento, giunto a Firenze nel 1204 con dentro la reliquia di San Filippo Apostolo, sia il reliquiario con cui la reliquia venne trasportata da Constantinopoli a Gerusalemme.

Le notevoli qualità della placchetta, nonostante l'aspetto non tanto raffinato, che soprattutto si vedono nella capacità dell'artista di conferire il lievo movimento alle membra sotto il manto, ci fanno supporre che essa sia espressione di un grande centro artistico dell'impero bizantino. Aggiungiamo inoltre che sembra impossibile che il braccio di San Filippo Apostolo fosse privo di una teca, quando si custodiva nel tesoro di Manuele Comneno, prima di essere dato alla sua nipote Maria che lo portò a Gerusalemme. Supponiamo infine che il forzierino con il quale giunse la reliquia da $\mathrm{Ge}$ rusalemme a Firenze, fosse lo stesso con cui fu portato da Constantinopoli a Gerusalemme e cosi sembra che all'opera debba essere attribuita un'origine constantinopolitana, anche se potrebbe restare in proposito qualche perplessità dovuta alla scarsa documentazione che abbiamo per l'arte bizantina del XII secolo e alla mancanza di una chiara definizione artistica nella storiografia delle peculiaritả delle varie scuole operanti all'interno delle regioni dell'impero bizantino.

GIOVANNI CANONIDIS

32. Mores et consuetudines..., cit., paragrafo 3 . 